\title{
TAPP or TEP? Population-Based Analysis of Prospective Data on 4,552 Patients Undergoing Endoscopic Inguinal Hernia Repair
}

\author{
Markus Gass • Vanessa M. Banz • Laura Rosella • \\ Michel Adamina • Daniel Candinas • \\ Ulrich Güller
}

Published online: 7 September 2012

(c) Société Internationale de Chirurgie 2012

\begin{abstract}
Background Whether total extraperitoneal inguinal hernia repair (TEP) is associated with worse outcomes than transabdominal preperitoneal inguinal hernia repair (TAPP) continues to be a matter of debate. The objective of this large cohort study is to compare outcomes between patients undergoing TEP or TAPP.

Methods Based on prospective data of the Swiss association of laparoscopic and thoracoscopic surgery, all patients undergoing unilateral TEP or TAPP between 1995 and 2006 were included. The following outcomes were compared: conversion rates, intraoperative and postoperative complications, duration of operation.

Results Data on 4,552 patients undergoing TEP $(n=3,457)$ and TAPP $(n=1,095)$ were collected prospectively. Average age and American Society of

This work was reported as a podium presentation at the Swiss Surgical Society annual meeting, June 2011, Geneva, Switzerland and as a poster presentation at the Swiss Visceral Surgery Society annual meeting, September 2011, Montreux, Switzerland.
\end{abstract}

M. Gass · V. M. Banz · D. Candinas · U. Güller ( $\bowtie)$ Department of Visceral Surgery and Medicine,

University Hospital Bern, CH-3010 Bern, Switzerland

e-mail: ulrich.guller@gmail.com

L. Rosella

Dalla Lana School of Public Health, University of Toronto,

Toronto, ON, Canada

\section{Adamina}

Department of Surgery, Cantonal Hospital St. Gallen,

St. Gallen, Switzerland

U. Güller

Department of Medical Oncology \& Hematology,

Cantonal Hospital St. Gallen, St. Gallen, Switzerland
Anesthesiologists score were similar in the two groups. Patients undergoing TEP had a significantly higher rate of intraoperative complications (TEP $1.9 \%$ vs. TAPP $0.9 \%$, $p=0.029$ ) and surgical postoperative complications (TEP: $2.3 \%$ vs. TAPP: $0.8 \%, p=0.003)$. The postoperative length of stay was longer for patients undergoing TAPP (2.9 vs. 2.3 days, $p=0.002$ ), whereas the duration of the operation was longer for TEP (66.6 vs. $59.0 \mathrm{~min}$, $p<0.001$ ) and the conversion rate was higher (TEP $1.0 \%$ vs. TAPP $0.2 \%, p=0.011$ ).

Conclusions This study is one of the first populationbased analyses comparing TEP and TAPP in a prospective cohort of more than 4,500 patients. Intraoperative and surgical postoperative complications were significantly higher in patients undergoing TEP. TEP is also associated with longer operating times and higher conversion rates. Therefore, on a population-based level, the TAPP technique appears to be superior to the TEP repair in patients undergoing unilateral inguinal hernia repair.

\section{Introduction}

Inguinal hernia is a common surgical problem, with more than 800,000 repairs in the United States per annum [1]. Over the past two decades, laparoscopic inguinal hernia repair has become more and more popular. Several surgical techniques have been developed over the past years, and total extraperitoneal (TEP) and transabdominal preperitoneal inguinal hernia repair (TAPP) are the endoscopic techniques that are most commonly used.

Widely accepted indications for endoscopic inguinal hernia repair are hernia recurrence, particularly following an initially performed open repair, and bilateral hernias [2]. Increasingly, however, endoscopic techniques are chosen 
as the primary treatment of choice for inguinal hernia as these techniques seem to be advantageous compared to conventional open repair owing to the smaller incisions with less postoperative pain and faster recovery [3].

With the TAPP technique, the mesh is placed between the abdominal wall and the peritoneum in the preperitoneal space. This method necessitates access to the abdominal cavity, which inevitably endures all the risks of an intraperitoneal approach, including involuntary injury of the abdominal organs. In contrast, the TEP technique creates a preperitoneal space without entering the abdominal cavity, although abdominal organs attached to the peritoneum by adhesions remain at potential risk of injury. Preparation in the preperitoneal space using the TEP technique is often depicted as being more complex, as anatomic landmarks are difficult to identify compared to the TAPP technique. This is reflected in the very large number of (approximately 200-300) interventions needed to achieve an acceptable threshold of complications and recurrence rates [4]. Although these numbers tend to be lower for the TAPP technique, both laparoscopic hernia repair methods have a longer learning curve than standard open repair $[5,6]$.

We have previously reported our data on unilateral and bilateral inguinal hernia repair using the TEP approach and found that bilateral TEP can be performed with outcomes similar to those achieved with unilateral TEP [7]. However, whether the outcomes of the TEP or the TAPP repair differ with regard to intraoperative or postoperative complications, length of postoperative hospital stay, duration of operation, or conversion rates remains unclear, and population-based data in literature are inconsistent.

The objective of the present investigation was to compare, on a population-based level, short-term outcomes after TEP versus TAPP in more than 4,500 patients undergoing inguinal hernia repair.

\section{Materials and methods}

The analysis was based on data from the Swiss Association of Laparoscopic and Thoracoscopic Surgery (SALTS) database, a prospective database of patients undergoing laparoscopic procedures in Switzerland.

Inclusion and exclusion criteria

Between 1995 and 2006 all patients aged $\geq 18$ years requiring elective unilateral TEP or TAPP for an inguinal hernia were included in the present study. Patients with bilateral or recurrent hernias were excluded. All data were prospectively collected and entered in a centralized database (Qualicare; Qualidoc, Liebefeld-Bern, Switzerland) by a data manager who works independently from the authors of this study. Missing values from the data sheets were obtained by the data manager. Baseline demographics were extracted, as were data regarding the following clinical outcomes: conversion rate; intraoperative and postoperative surgical complications (e.g., wound infection, hematoma); general postoperative complications (e.g., pneumonia, urinary tract infection, pulmonary embolism); length of operation; postoperative length of hospital stay (LOS).

Statistical analysis

All statistical computations were carried out by an experienced statistician (L.R.). Outcomes were summarized using the mean or median and standard deviation (for continuous variables) or proportions (for categorical variables). For continuous outcomes, mean differences between TAPP versus TEP were tested using a two-sample $t$ test. Categorical variables and rates were tested for differences using a $\chi^{2}$ test. Multivariable-adjusted analyses were performed on the following outcomes: intraoperative complications, postoperative surgical complications (e.g., bleeding, surgical site infection), general postoperative complications (e.g., pneumonia, urinary tract infection), conversion rates, postoperative LOS, operating time. Complications and conversion rate outcomes were modeled using logistic regression. For intraoperative and postoperative complications, the event of the univariate and multivariable analysis for each outcome was defined as the presence of one or more complications. Duration of operation was dichotomized such that the outcome was defined as being in the upper quartile (above the 75th percentile) of the distribution. Postoperative LOS was modeled using a linear regression analysis. All multivariable models were adjusted for age, sex, and American Society of Anesthesiologists (ASA) score. A $p$ value of $\leq 0.05$ was considered statistically significant. All statistical tests were two-sided, and all statistical analyses were performed using SAS version 9.1 (SAS Institute, Cary, NC, USA).

\section{Results}

Between 1995 and 2006, a total of 4,552 patients underwent elective unilateral TEP $(n=3,457)$ or TAPP ( $n=1,095)$ for inguinal hernia. Of these, 4,233 (93\%) patients were male $(94.6 \%$ in the TEP group, $88.0 \%$ in the TAPP group). Mean age in the TEP group was 53.6 years and 54.9 years in the TAPP group $(p=0.009)$. Mean ASA score was 1.4 for both groups. Baseline demographics are provided in Table 1.

Patients undergoing TEP had a statistically significant increased rate of intraoperative complications (TEP $1.9 \%$ vs. TAPP $0.9 \% ; p=0.029)$ and postoperative surgical complications (TEP $2.3 \%$ vs. TAPP $0.8 \% ; p=0.003$ ). 
Table 1 Patient characteristics $(n=4,552)$

\begin{tabular}{lll}
\hline Variable & TEP $(n=3,457)$ & TAPP $(n=1,095)$ \\
\hline Age (years), mean \pm SD & $53.6 \pm 14.3$ & $54.9 \pm 15.3$ \\
ASA score, mean \pm SD & $1.4 \pm 0.6$ & $1.4 \pm 0.6$ \\
Male sex & $3,271(94.6 \%)$ & $964(88.0 \%)$ \\
\hline
\end{tabular}

ASA American society of anesthesiologists; TEP total extraperitoneal inguinal hernia repair; TAPP transabdominal preperitoneal inguinal hernia repair

General postoperative complications were not statistically different between the two methods (TEP $0.7 \%$ vs. TAPP $0.4 \% ; p=0.195)$. Intraoperative and postoperative mortality was zero in both groups. The average duration of the operation was longer for patients undergoing TEP than for those with TAPP (66.6 vs. 59 min, respectively; $p<0.001$ ), and conversion rates were higher (TEP $1.0 \%$ vs. TAPP $0.2 \% ; p=0.011$ ). Postoperative LOS was significantly longer for patients undergoing TAPP than for those with TEP ( 2.9 vs. 2.3 days, $p=0.002$ ).

Unadjusted and risk-adjusted comparisons of the various outcomes analyzed are displayed in Tables 2 and 3, respectively.

\section{Discussion}

The findings from the present study demonstrate that both endoscopic hernia repair methods, TAPP and TEP, are safe, feasible, and associated with a low postoperative morbidity rate for the repair of primary unilateral inguinal hernia. However, on a population-based level (sample size of more than 4,500 patients), TAPP is statistically superior to TEP for all evaluated variables except postoperative LOS and general postoperative complications. For the individual patient, however, these differences are likely to be of minor relevance as reflected in the high numbers needed to treat (Table 2).
A systematic Cochrane database review from 2005 comparing TAPP to TEP was unable to provide a conclusive answer with regard to the superiority of either technique owing to the lack of reliable data [8], with only one randomized controlled trial [9] and nine other nonrandomized observational studies included in the analyses [10-17]. Also, the randomized controlled trial conducted by Schrenk et al. [9] is limited by the small patient numbers: TAPP, $n=28$; TEP, $n=24$. In their investigation, as in our study, operating times were slightly longer in the TEP group.

A recent study from China by Gong et al. [18] compared the open tension-free mesh plug technique with the TAPP and TEP techniques for primary unilateral inguinal hernia repair based on 164 patients. In their study, operating times were decidedly longer (79 $\mathrm{min}$ for TEP, $76 \mathrm{~min}$ for TAPP) than in our patient cohort. A subanalysis of this investigation revealed no significant differences between TAPP and TEP regarding duration of operation, intraoperative bleeding, postoperative pain, complications, recurrence, and LOS or recovery. Our reported postoperative complication rates of well below $5 \%$ are comparable to most studies in the literature $[19,20]$, although they are much lower compared to those reported in the Gong study $(12.0 \%$ for the TAPP group and $13.5 \%$ for the TEP group) [18].

Contrary to our current data, a large study by Felix et al. [11] from the mid-1990s showed significantly more major

Table 2 Comparison of unadjusted outcomes between patients undergoing TEP versus TAPP

\begin{tabular}{lllll}
\hline Variable & TEP $(n=3,457)$ & TAPP $(n=1,095)$ & $p$ & Number needed to treat \\
\hline $\begin{array}{l}\text { Intraoperative complications } \\
\text { Postoperative complications }\end{array}$ & $65(1.9 \%)$ & $10(0.9 \%)$ & 0.029 & 100 \\
$\quad$ Surgical $^{\mathrm{a}}$ & $78(2.3 \%)$ & $9(0.8 \%)$ & 0.003 & 67 \\
General $^{\mathrm{b}}$ & $25(0.7 \%)$ & $4(0.4 \%)$ & 0.195 & 333 \\
Conversion to open procedure $^{\text {Duration of operation (min), mean } \pm \text { SD }}$ & $33(1.0 \%)$ & $2(0.2 \%)$ & 0.011 & 125 \\
Postoperative LOS (days), mean \pm SD & $66.6 \pm 31.0$ & $59.0 \pm 27.0$ & $<0.0001$ & NA \\
\hline
\end{tabular}

$L O S$ length of stay; NA not applicable

${ }^{a}$ Defined as being directly related to the surgical procedure (e.g., wound infection, hematoma)

${ }^{\mathrm{b}}$ Defined as not being directly related to the surgical procedure (e.g., pneumonia, urinary tract infection, pulmonary embolism) 
Table 3 Comparison of risk-adjusted outcomes between patients undergoing TEP and TAPP

\begin{tabular}{llrr}
\hline Variable & Odds ratio & LCL & UCL \\
\hline Complications (TEP vs. TAPP) & & & $p$ \\
$\quad$ Intraoperative & 2.028 & 1.034 & 3.979 \\
Postoperative & & & 0.04 \\
$\quad$ Surgical & 2.715 & 1.353 & 5.447 \\
$\quad$ General & 2.144 & 0.738 & 6.225 \\
Conversion to open procedure (TEP vs. TAPP) & 5.235 & 1.25 & 0.005 \\
Length of operation (TEP vs. TAPP) & 1.009 & 1.007 & 0.161 \\
Postoperative LOS (TEP vs. TAPP) & 0.932 & 0.898 & 0.024 \\
\hline
\end{tabular}

UCL upper $95 \%$ confidence limit; $L C L$ lower $95 \%$ confidence limit

complications in the TAPP group than in the TEP group. A total of 866 patients underwent 1,115 endoscopic hernioplasties (733 TAPP, 382 TEP) with 11 patients suffering major complications (two recurrences, six trocar hernias, one small-bowel obstruction, one trocar injury one dissection injury of the small bowel) in the TAPP group compared to only one recurrence and no intraperitoneal complications in the TEP group. With recovery time not differing between the both groups, the authors advocate that TEP repair is superior to TAPP. It is crucial, however, to interpret this study with caution as the procedures were performed during the mid-1990s when both techniques were still new and many surgeons were at the beginning of their learning curve.

In our study unadjusted and risk-adjusted analyses of conversion rates revealed significantly higher rates for the TEP group, as is reflected by a high odds ratio $(>5)$. The finding of a higher conversion rate in the TEP group can be explained by the easier identification of anatomic structures and landmarks with the TAPP technique. The odds ratio of five was nevertheless unexpected. Interestingly, conversion rates in the literature are higher for the TEPP technique than in our investigation. Cohen et al. [10] and Van Hee et al. [14] reported conversion rates of $4 \%$ and $7 \%$, respectively. The rates are much higher than in our group, again probably because these studies result from the late 1990s, when the TEP technique was rather new.

\section{Strengths and limitations}

The main limitation of our study is the lack of long-term outcomes, including data on the incidence of hernia recurrence, long-term pain, or degree of patient satisfaction with TEP or TAPP. Furthermore, the SALTS data set does not provide accurate information on the grading (severity) of the complications.

Our study has a number of strengths. First, the large sample size confers a high degree of statistical power for detecting relevant differences. Second, the data were gathered prospectively and queried for incomplete values, so it has virtually no missing data. Finally, although we examined only patients treated in Switzerland, we believe the results of this population-based study can be extrapolated to other countries with high standards of laparoscopic surgical technique [21, 22].

\section{Conclusions}

To our knowledge, this study is among the first populationbased analyses comparing outcomes of patients undergoing TEP and TAPP in a large prospective cohort. Intraoperative and surgical postoperative complications were significantly higher in patients undergoing TEP. Moreover, TEP is associated with longer operating times and higher conversion rates. Therefore, on a population-based level, the TAPP technique appears to be superior to the TEP repair in patients undergoing unilateral inguinal hernia repair.

Acknowledgments This study was supported by grants from Johnson \& Johnson and Covidien.

Conflict of interest Drs. Markus Gass, Vanessa Banz, Laura Rosella, Michel Adamina, Daniel Candinas, and Ulrich Güller have no conflicts of interest or financial ties to disclose.

\section{References}

1. Rutkow IM (2003) Demographic and socioeconomic aspects of hernia repair in the United States in 2003. Surg Clin North Am 83:1045-1051, v-vi

2. Wauschkuhn CA, Schwarz J, Boekeler U et al (2010) Laparoscopic inguinal hernia repair: gold standard in bilateral hernia repair? Results of more than 2800 patients in comparison to literature. Surg Endosc 24:3026-3030

3. Grant AM (2002) Laparoscopic versus open groin hernia repair: meta-analysis of randomised trials based on individual patient data. Hernia 6:2-10

4. Zendejas B, Onkendi EO, Brahmbhatt RD et al (2011) Long-term outcomes of laparoscopic totally extraperitoneal inguinal hernia 
repairs performed by supervised surgical trainees. Am J Surg 201:379-383 discussion 383-374

5. Wright D, O'Dwyer PJ (1998) The learning curve for laparoscopic hernia repair. Semin Laparosc Surg 5:227-232

6. Lal P, Kajla RK, Chander J et al (2004) Laparoscopic total extraperitoneal (TEP) inguinal hernia repair: overcoming the learning curve. Surg Endosc 18:642-645

7. Gass M, Rosella L, Banz V et al (2012) Bilateral total extraperitoneal inguinal hernia repair (TEP) has outcomes similar to those for unilateral TEP: population-based analysis of prospective data of 6,505 patients. Surg Endosc 26:1364-1368

8. McCormack K, Wake BL, Fraser C et al (2005) Transabdominal pre-peritoneal (TAPP) versus totally extraperitoneal (TEP) laparoscopic techniques for inguinal hernia repair: a systematic review. Hernia 9:109-114

9. Schrenk P, Woisetschlager R, Rieger R et al (1996) Prospective randomized trial comparing postoperative pain and return to physical activity after transabdominal preperitoneal, total preperitoneal or Shouldice technique for inguinal hernia repair. Br J Surg 83:1563-1566

10. Cohen RV, Alvarez G, Roll S et al (1998) Transabdominal or totally extraperitoneal laparoscopic hernia repair? Surg Laparosc Endosc 8:264-268

11. Felix EL, Michas CA, Gonzalez MH Jr (1995) Laparoscopic hernioplasty: TAPP vs TEP. Surg Endosc 9:984-989

12. Khoury N (1995) A comparative study of laparoscopic extraperitoneal and transabdominal preperitoneal herniorrhaphy. J Laparoendosc Surg 5:349-355

13. Lepere M, Benchetrit S, Debaert M et al (2000) A multicentric comparison of transabdominal versus totally extraperitoneal laparoscopic hernia repair using PARIETEX meshes. J Soc Laparoendosc Surg 4:147-153
14. Van Hee R, Goverde P, Hendrickx L et al (1998) Laparoscopic transperitoneal versus extraperitoneal inguinal hernia repair: a prospective clinical trial. Acta Chir Belg 98:132-135

15. Baca I, Schultz C, Gotzen V et al (eds) (2000) Laparoscopic inguinal hernia repair: a review of 2500 cases. Monduzzi Editore, Bologna

16. Tamme C, Scheidbach H, Hampe C et al (2000) Totally extraperitoneal endoscopic inguinal hernia repair (TEP). Surg Endosc 17:190-195

17. Leibl BJ, Schmedt CG, Kraft K (2000) Laparoscopic transperitoneal hernioplasty (TAPP): efficiency and dangers. Chir Gastroenterol 16:106-109

18. Gong K, Zhang N, Lu Y et al (2011) Comparison of the open tension-free mesh-plug, transabdominal preperitoneal (TAPP), and totally extraperitoneal (TEP) laparoscopic techniques for primary unilateral inguinal hernia repair: a prospective randomized controlled trial. Surg Endosc 25:234-239

19. Perko Z, Rakic M, Pogorelic Z et al (2011) Laparoscopic transabdominal preperitoneal approach for inguinal hernia repair: a five-year experience at a single center. Surg Today 41:216-221

20. Dulucq JL, Wintringer P, Mahajna A (2009) Laparoscopic totally extraperitoneal inguinal hernia repair: lessons learned from 3,100 hernia repairs over 15 years. Surg Endosc 23:482-486

21. Guller U, Rosella L, Karanicolas PJ et al (2010) Population-based trend analysis of 2813 patients undergoing laparoscopic sigmoid resection. Br J Surg 97:79-85

22. Brugger L, Rosella L, Candinas D et al (2011) Improving outcomes after laparoscopic appendectomy: a population-based, 12-year trend analysis of 7446 patients. Ann Surg 253:309-313 